\title{
Concentraciones séricas de triyodotironina, tiroxina y perfil lipídico en bovinos lecheros alimentados con una ración con bajo contenido de selenio ${ }^{\#}$
}

\author{
Serum concentrations of triiodothyronine, thyroxine and lipid profile \\ in dairy cows fed with a low selenium diet ${ }^{\#}$

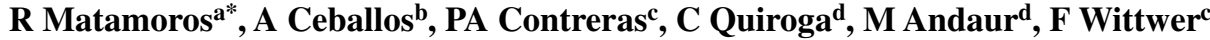
${ }^{a}$ Escuela de Medicina Veterinaria, Universidad Santo Tomás. Temuco, Chile.

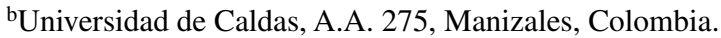
Dirección actual: University of Prince Edward Island. Charlottetown, PEI C1A 4P3, Canada.
'Instituto de Ciencias Clínicas Veterinarias, Universidad Austral de Chile, Valdivia, Chile.
${ }^{\mathrm{d} E s c u e l a ~ d e ~ M e d i c i n a ~ V e t e r i n a r i a, ~ U n i v e r s i d a d ~ C a t o ́ l i c a ~ d e ~ T e m u c o . ~ T e m u c o, ~ C h i l e . ~}$

\begin{abstract}
SUMMARY
The aim of this study was to evaluate the effect of a low Selenium diet and its supplementation on the serum levels of $\mathrm{T}_{3}, \mathrm{~T}_{4}$ and on the lipid profile in dairy cows under an induced oxidative stress. Twelve pregnant dairy Chilean-Friesian cows were selected and assigned to one of two groups, control $\left(n=6\right.$, Se-D) and treated $\left(n=6\right.$, Se-S) with barium selenate $\left(\mathrm{BaSeO}_{4}\right)$, both receiving a Selenium-deficient diet (based on a $0.03 \mathrm{ppm} \mathrm{Se-content} \mathrm{of}$ the DM in the diet before calving, and $0.05 \mathrm{ppm}$ after calving) and oxidized tallow ( $0.5 \mathrm{~kg} / \mathrm{cow} / \mathrm{day})$. The Se-S group was supplemented with selenium $(1 \mathrm{mg} / \mathrm{kg})$ in the form of $\mathrm{BaSeO}_{4}(1 \mathrm{~mL} / 50 \mathrm{~kg}$, s.c.). Prior to the supplementation and every 15 days, heparinized blood and plain blood samples were taken by venous puncture. The blood activity of glutathione peroxidase (GSH-Px, EC 1.15.1.9) was evaluated, also the serum concentrations of $\mathrm{T}_{3}, \mathrm{~T}_{4}$, total cholesterol, lipoproteins (HDL, LDL and VLDL) and triacylglycerols (TAG). The differences were settled by means of a PROC MIXED of SAS, and the option REPEATED, significance was set at $\mathrm{P}<0.05$. The Se-supplementation with $\mathrm{BaSeO}_{4}$ did not produce changes in the milk yield $(\mathrm{P}>0.05)$, and the blood activity of GSH-Px was higher in the group Se-S starting 45 days after supplementation $(\mathrm{P}<0.05)$, while the activity in the group Se-D was lower along the study $(\mathrm{P}<0.05)$. There were no differences for $\mathrm{T}_{3}$ and $\mathrm{T}_{4}(\mathrm{P}>0.05)$. Serum concentration of cholesterol, $\mathrm{LDL}, \mathrm{VLDL}$ and TAG were similar between groups $(\mathrm{P}>0.05)$, there was a tendency to a higher concentration of HDL in the non-supplemented group $(\mathrm{P}=0.047)$. The results suggested that a low Se-diet does not induce changes in either the thyroid function or the serum concentration of cholesterol and lipoproteins, except for the concentration of HDL, being further studies in dairy cows necessary.
\end{abstract}

Palabras clave: selenio, lípidos, hormonas tiroideas.

Key words: selenium, lipids, thyroid hormones.

\section{INTRODUCCION}

La deficiencia de selenio (Se) se ha asociado con algunas patologías del bovino como enfermedad del músculo blanco, retención de placenta, inmunosupresión y mastitis, entre otras (Miller y col 1993); igualmente, alteraciones en el crecimiento y desarrollo estarían relacionadas con cambios en el metabolismo hormonal tiroideo inducidos por la deficiencia de Se (Wichtel y col 1996a, Wichtel y col 1996 b).

El yodo, zinc y Se participan en la síntesis de las hormonas tiroideas (Ruz y col 1999). Además, el Se forma parte de la estructura de diferentes proteínas del organismo y su concentración en la glándula tiroides es más alta que en otros tejidos (Howie y col 1995); también, es constituyente de cuatro glutatión peroxidasas y tres iodotironina

Aceptado: 13.03.2007

\# Proyecto FONDECYT-Chile 1990993.

*rmatamoros@ust.cl 5'-deiodinasas (DI; EC 3.8.1.4), tipos I, II y III, entre otras selenoproteínas (Wichtel 1998, Holben y Smith 1999). La DI tipo I se expresa en diferentes órganos, como el hígado, riñones y glándula tiroides, la tipo II lo hace en el sistema nervioso y el tejido adiposo pardo, y la tipo III en el útero y la placenta (Wichtel 1998, Allan y col 1999, Arthur y col 1999, Holben y Smith 1999).

Las DI catalizan la deiodinación de la prohormona 3,5,3',5'-tetrayodotironina (L-tiroxina, $\mathrm{T}_{4}$ ) a su forma metabólicamente activa la 3,5,3'-triyodotironina $\left(\mathrm{T}_{3}\right)$ (Berry y Larsen 1992, Brent 1994, Arthur y col 1999). Las hormonas tiroideas tienen como efecto el aumento de la tasa metabólica celular. En particular sobre el metabolismo lipídico, la actividad tiroidea es inversa a la concentración sérica de colesterol, lo que estaría indicando que una hipercolesterolemia podría asociarse con alteraciones de la función tiroidea; pero, una hipocolesterolemia tiene poco valor como indicador de hipertiroidismo (Brent 1994, Duntas 2002).

En rebaños lecheros del sur de Chile se ha descrito la deficiencia de Se diagnosticada mediante la determinación 
de la actividad eritrocitaria de la enzima antioxidante glutatión peroxidasa (GSH-Px; EC 1.11.1.9) (Ceballos y col 1998, Wittwer y col 2002). En vacas lecheras mantenidas en praderas con forrajes con un bajo contenido de Se, las concentraciones de $\mathrm{T}_{3}$ se mantuvieron significativamente más bajas durante la lactancia que en vacas suplementadas con Se (Contreras y col 2002, 2005). También se han descrito signos clínicos compatibles con una deficiencia de Se en caprinos Cashmere, habiéndose observado una respuesta positiva a la suplementación con Se, considerándose el cambio de peso como indicador (Ceballos y col 1999b); sin embargo, no se observaron diferencias en la concentración de $\mathrm{T}_{4}$ entre las cabras suplementadas y no suplementadas con Se (Ceballos y col 1999 ab); pero, no se estudió el efecto sobre colesterol, lipoproteínas y triacilgliceroles.

En consideración a lo señalado, el objetivo de este estudio fue evaluar en vacas en lactancia sometidas a un estrés oxidativo inducido, el efecto de una dieta con un bajo contenido de Se y su suplementación sobre las concentraciones séricas de las hormonas $\mathrm{T}_{3}$ y $\mathrm{T}_{4}, \mathrm{y}$ sobre las concentraciones de colesterol, lipoproteínas y triacilgliceroles.

\section{MATERIAL Y METODOS}

Animales seleccionados y grupos experimentales. En este estudio se trabajó con doce vacas Frisón Negro Chileno, clínicamente sanas, gestantes (siete meses) y con una edad promedio de cinco años, las que fueron asignadas a uno de dos grupos con seis vacas cada uno. Los grupos fueron homogéneos en cuanto a la edad de las vacas, número de partos, peso, condición corporal y producción de leche según el registro de la lactancia previa al estudio. Ambos grupos recibieron una ración con bajo contenido de $\mathrm{Se}$, manteniéndose un grupo durante todo el estudio con esta ración (Se-D) y el otro grupo recibió vía subcutánea una dosis única de $\mathrm{Se}$ (Se-S) aproximadamente 45 días antes del parto. Los animales no recibieron ningún tratamiento que pudiera afectar las concentraciones séricas de $\mathrm{T}_{3}, \mathrm{~T}_{4} \mathrm{O}$ los lípidos séricos estudiados, tales como glucocorticoides o sulfonamidas.

Alimentación. Las vacas se mantuvieron desde seis semanas antes del parto en cubículos con comederos individuales, en piso de concreto y con cama de paja de trigo. Durante el preparto cada vaca recibió diariamente $9,5 \mathrm{~kg}$ de heno con un bajo contenido de Se $(0,02 \mathrm{ppm}$ base materia seca) y un $\mathrm{kg}$ de un concentrado comercial $\left(\right.$ Cosetan $^{\circledR}$, Biomaster-IANSA, Chile) con un contenido de Se de 0,12 ppm base materia seca. Al momento del parto las vacas permanecieron tres días con sus crías antes de empezar su ordeño mecánico dos veces por día. Las vacas recibieron durante la lactancia $11,5 \mathrm{~kg}$ de heno, $0,5 \mathrm{~kg}$ de afrecho de soya (Se: $<0,17 \mathrm{ppm}$ base materia seca), $0,15 \mathrm{~kg}$ de sales minerales $\sin$ Se y $0,5 \mathrm{~kg}$ de sebo oxidado para alimentación animal (Se: $<0,1$ ppm base materia seca) desde 15 días postparto y durante todo el período de estudio. El sebo oxidado se ofreció con el objeto de inducir un ambiente propio de estrés oxidativo. Además hasta $5 \mathrm{~kg}$ de Cosetan ${ }^{\circledR}$ y hasta $0,12 \mathrm{~kg}$ de urea, tratando de satisfacer los requerimientos y preservando mantener una ración con un bajo contenido de Se. El máximo consumo de Se durante el período previo al parto fue $0,03 \mathrm{mg} / \mathrm{kg}$ base materia seca, y durante la lactancia $0,05 \mathrm{mg} / \mathrm{kg}$ base materia seca. El consumo de alimento se controló diariamente pesando la cantidad ofrecida y el eventual alimento no consumido. La condición corporal promedio al parto para las vacas Se-S fue 3,0, mientras que en el grupo Se-D fue 2,90 al mes posparto el valor fue más bajo llegando a 2,1 y 2,0 para las vacas Se-S y Se-D, respectivamente. El agua se mantuvo ad libitum y la leche se pesó diariamente hasta finalizar el estudio.

Suplementación con Se y obtención de muestras. El grupo suplementado recibió $1 \mathrm{mg}$ de $\mathrm{Se} / \mathrm{kg} / \mathrm{pv}$ usando selenato de bario $\left(\mathrm{BaSeO}_{4}\right)\left(\right.$ Deposel $^{\circledR}$, Young Animal Health Ltd., New Zealand) en dosis única de $1 \mathrm{~mL} / 50 \mathrm{~kg} / \mathrm{pv}$, mientras que las vacas del grupo Se-D no recibieron suplementación.

De cada vaca se obtuvieron simultáneamente dos muestras de sangre (con y sin heparina) mediante venopunción coccígea, en el período previo al parto y a la suplementación (45 días antes del parto) y luego en la lactancia cada 15 días hasta 105 días postparto. La muestra de sangre con heparina, previa determinación de la concentración de hemoglobina, fue hemolizada con un diluyente comercial (Ransel diluyente ${ }^{\circledR}$, Randox Lab., Irlanda del Norte) y se mantuvo a $-20^{\circ} \mathrm{C}$ hasta el análisis de la actividad sanguínea de GSH-Px; igualmente, el suero se mantuvo a una temperatura similar hasta el análisis de la concentración de las hormonas $\mathrm{T}_{3} \mathrm{y} \mathrm{T}_{4}$, colesterol, lipoproteínas de alta (HDL), baja (LDL) y muy baja densidad (VLDL) y triacilgliceroles (TAG).

Análisis. El balance nutricional de Se fue evaluado mediante la actividad sanguínea de GSH-Px en un hemolizado de las muestras de sangre tomadas con heparina en ambos grupos de vacas. Se empleó un reactivo comercial (Ransel ${ }^{\circledR}$, Randox Lab., Irlanda del Norte) basado en una técnica cinética compuesta NADPH dependiente, según la modificación del método descrito por Paglia y Valentine en 1967 (Ceballos y col 1998 a). Las lecturas se realizaron en un espectrofotómetro Hitachi 4020 (ROCHE Diagnostics GmbH, Mannheim, Alemania) y la actividad se expresó en U/g Hb e interpretada según Ceballos y Wittwer (1996) y Ceballos y col $\left(1998^{\mathrm{a}}\right)$, quienes señalan que los valores inferiores a $60 \mathrm{U} / \mathrm{g} \mathrm{Hb}$ se consideran deficientes, entre 61 y $100 \mathrm{U} / \mathrm{g} \mathrm{Hb}$ como bajo-marginal, entre 101 y $130 \mathrm{U} / \mathrm{g}$ $\mathrm{Hb}$ marginales y sobre $130 \mathrm{U} / \mathrm{g} \mathrm{Hb}$ son adecuados. Las concentraciones séricas de $\mathrm{T}_{3} \mathrm{y} \mathrm{T}_{4}$ se midieron mediante un inmunoensayo utilizando la técnica de electroquimioluminescencia en un equipo Elecsys $1010^{\circledR}$ (ROCHE 
Diagnostics GmbH, Mannheim, Alemania), los resultados se expresan en nmol/L. Además se determinaron las concentraciones séricas de colesterol (método enzimático CHOD-PAP), lipoproteínas HDL (método fosfotungstato magnesio CHOD-PAP), LDL (ecuación de Friedewald), VLDL (método enzimático GPO-PAP) y TAG (método enzimático GPO-PAP). Los análisis se realizaron mediante espectrofotometría UV usando reactivos comerciales (ROCHE Diagnostics GmbH, Mannheim, Alemania) y los resultados se expresaron en $\mathrm{mmol} / \mathrm{L}$.

El análisis del contenido de selenio se realizó mediante espectrometría de masa con plasma inductivamente acoplado (ICP-MS), previa digestión de la materia orgánica de la muestra con ácido nítrico y perclórico. Los análisis se realizaron en los Laboratorios Hill en Nueva Zelanda.

Análisis estadístico. El diseño correspondió a un estudio longitudinal completamente al azar. Las diferencias entre grupos y entre períodos se establecieron mediante una prueba de análisis de varianza (ANDEVA) para un modelo jerárquico/parcelas-divididas (split-plot) según la ecuación:

$$
Y_{i j}=\mu+\alpha_{\mathrm{ttment}(i)}+\beta_{j}+\alpha \beta_{\mathrm{ttment}(i), j}+A_{i}+\varepsilon_{i j}
$$

En donde:

$Y_{i j}=$ GSH-Px en la $i$-ésima vaca al tiempo $j$.

$\mu=$ media general.

$\alpha_{\text {ttment }(i)}=$ efecto del tratamiento en la $i$-ésima vaca.

$\beta_{j}=$ efecto de la $j$-ésima observación en la $i$-ésima vaca.

$\alpha \beta_{\mathrm{ttment}(i), j}=$ interacción.

$A_{i}=$ efecto aleatorio de la $i$-ésima vaca.

$\varepsilon_{i j}=$ error.

Posteriormente se realizó una prueba de comparación múltiple de Bonferroni, ajustándose el nivel de significancia según el número de comparaciones. El análisis se efectuó usando el PROC MIXED de SAS (SAS Inst. Inc. Cary, NC, USA) empleando también la opción REPEATED para incluir la matriz de varianzas-covarianzas entre medidas repetidas en un mismo individuo.

\section{RESULTADOS}

Glutatión peroxidasa. La actividad sanguínea de GSH-Px observada en las vacas que recibieron la ración baja en Se (Se-D) disminuyó a lo largo del estudio con respecto a los valores observados en el grupo Se-S $(\mathrm{P}<0,05)$, observándose un valor basal de $111 \pm 21 \mathrm{U} / \mathrm{g} \mathrm{Hb}$, valor considerado bajo-marginal a los 30 días de la suplementación $(89 \pm 13 \mathrm{U} / \mathrm{g} \mathrm{Hb})$ y al final del estudio $(65 \pm 8 \mathrm{U} / \mathrm{g}$ $\mathrm{Hb}$ ). En el grupo Se-S la actividad sanguínea de GSH-Px aumentó desde $119 \mathrm{U} / \mathrm{g}$ Hb previo a la suplementación hasta $300 \mathrm{U} / \mathrm{g} \mathrm{Hb}$ al final del período experimental $(\mathrm{P}<0,05$, figura 1$)$.

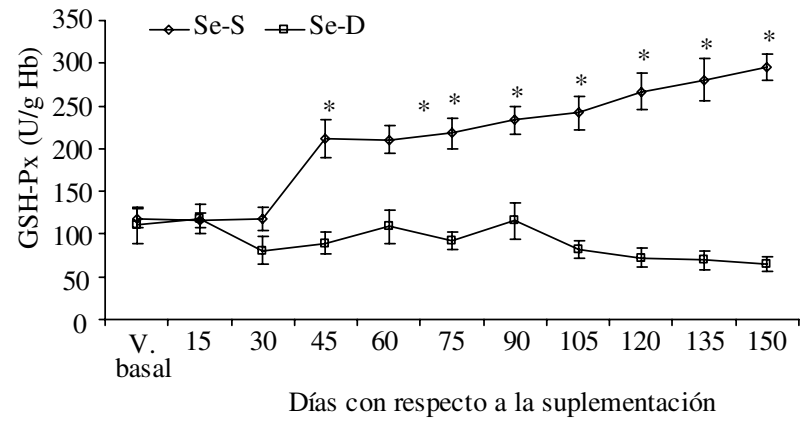

Figura 1. Promedio y error estándar ( $(\overline{\mathrm{X}} \pm \mathrm{EE})$ para la actividad de glutatión peroxidasa (GSH-Px) en vacas lecheras alimentadas con una ración baja en selenio (Se-D) o suplementadas con selenato de bario (Se-S).

*Diferencias significativas entre los grupos $(\mathrm{P}<0,05)$.

Mean and standard error ( $(\overline{\mathrm{X}} \pm \mathrm{SE})$ of the activity of glutathione peroxidase (GSH-Px) in dairy cows fed with a Se-deficient (Se-D) diet or supplemented (Se-S) with barium selenate $(\mathrm{Se}-\mathrm{S})$.

*Significant differences between groups $(\mathrm{P}<0.05)$.

Hormonas tiroideas. El promedio de las concentraciones séricas basales para $\mathrm{T}_{3}$ fue 1,8 $\pm 0,2 \mathrm{nmol} / \mathrm{L}$ y $1,6 \pm 0,2 \mathrm{nmol} / \mathrm{L}$ para las vacas Se-D y Se-S respectivamente $(\mathrm{P}>0,05)$. La concentración de $\mathrm{T}_{3}$ se redujo, encontrándose la concentración más baja durante la lactancia $(1,4 \pm 0,1 \mathrm{nmol} / \mathrm{L})$, entre 45 y 60 días después de iniciado el ensayo $(\mathrm{P}<0,05)$, no observándose diferencias según el tratamiento en los diferentes períodos de muestreo $(\mathrm{P}>0,05)$, posteriormente los valores se incrementaron hasta el final del estudio $(\mathrm{P}<0,05)$.

Las concentraciones séricas basales de $\mathrm{T}_{4}$ presentaron diferencias significativas entre los grupos Se-D y Se-S, los valores fueron $84,3 \pm 2,2 \mathrm{nmol} / \mathrm{L}$ y $103,0 \pm 9,7 \mathrm{nmol} / \mathrm{L}$ para Se-D y Se-S respectivamente $(\mathrm{P}<0,05)$. Estas concentraciones disminuyeron en ambos grupos $(\mathrm{P}<0,05)$ dos semanas después, valores que se mantuvieron bajos hasta 60 días después de iniciado el estudio y posteriormente se incrementaron sin alcanzar valores similares a los basales (figura 2). No se observaron diferencias en la concentración de $\mathrm{T}_{4}$ entre los grupos Se-D y Se-S en los diferentes períodos de muestreo $(\mathrm{P}>0,05)$.

Perfil lipídico. El promedio de las concentraciones basales de colesterol fue similar $(\mathrm{P}>0,05)$ entre los grupos Se-D $(3,7 \pm 0,2 \mathrm{mmol} / \mathrm{L})$ y $\mathrm{Se}-\mathrm{S}(3,7 \pm 0,1 \mathrm{mmol} / \mathrm{L})$, valores que declinaron significativamente dos semanas después $(\mathrm{P}<0,05)$, iniciándose posteriormente un incremento hasta 75 días después de iniciado el estudio (figura 3). El consumo de una dieta baja en Se no produjo cambios significativos en la concentración promedio de colesterol entre ambos grupos $(\mathrm{P}>0,05)$.

Previo al momento de suplementar las vacas, las concentraciones de HDL fueron similares entre los grupos estudiados $(\mathrm{P}>0,05)$, disminuyendo dos semanas después en ambos grupos (figura 3) e incrementándose a 


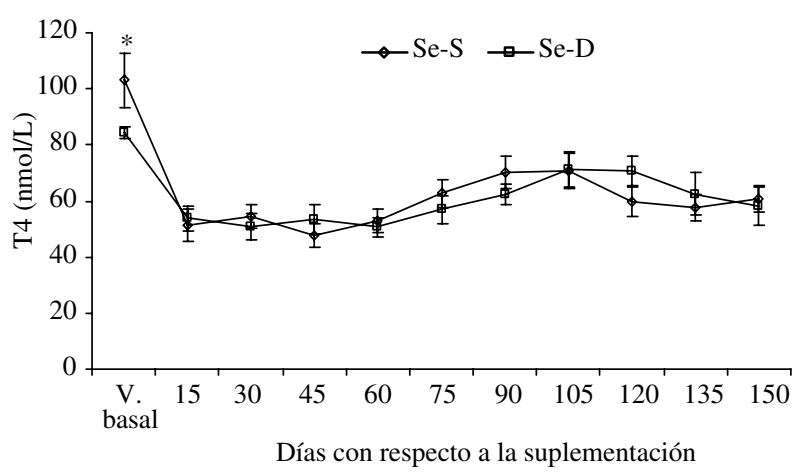

Figura 2. Promedio y error estándar $(\overline{\mathrm{X}} \pm \mathrm{EE})$ para la concentración sérica de tiroxina $\left(\mathrm{T}_{4}\right)$ en vacas lecheras alimentadas con una ración baja en selenio (Se-D) o suplementadas con selenato de bario (Se-S).

*Diferencias significativas entre los grupos $(\mathrm{P}<0,05)$.

Mean and standard error $(\overline{\mathrm{X}} \pm \mathrm{SE})$ of the serum thyroxine $\left(\mathrm{T}_{4}\right)$ concentration in dairy cows fed with a Se-deficient (Se-D) diet or supplemented ( $\mathrm{Se}-\mathrm{S}$ ) with barium selenate ( $\mathrm{Se}-\mathrm{S}$ ).

*Significant differences between groups $(\mathrm{P}<0.05)$.

partir de este momento $(\mathrm{P}<0,05)$. A lo largo del estudio se observó una tendencia a una concentración mayor en el grupo Se-D $(3,4 \pm 0,2 \mathrm{mmol} / \mathrm{L})$, mientras que para el grupo Se-S el promedio encontrado fue 3,0 $\pm 0,1 \mathrm{mmol} / \mathrm{L}$ $(\mathrm{P}=0,047)$.

Las concentraciones de LDL disminuyeron hasta el día 15 después de iniciado el estudio, posteriormente aumentaron en forma significativa $(\mathrm{P}<0,05$, figura 3$)$ en ambos grupos. No se observaron diferencias en la concentración de LDL entre ambos grupos Se-S y Se-D en los períodos estudiados $(\mathrm{P}>0,05)$.

Las concentraciones para las lipoproteínas VLDL y las concentraciones para TAG no presentaron diferencias entre los grupos Se-D y Se-S (P > 0,05), observándose sólo variaciones entre los diferentes períodos del estudio $(\mathrm{P}<0,05)$.

Producción de leche. La producción promedio de leche en el grupo Se-D fue 13,8 $3 \mathrm{~kg} /$ día y $14,0 \pm 3 \mathrm{~kg} / \mathrm{día}$ en el grupo Se-S $(\mathrm{P}>0,05)$. La máxima producción se alcanzó en la cuarta semana postparto, siendo similares las producciones entre los grupos $(\mathrm{P}>0,05)$.

\section{DISCUSION}

Las dietas con un bajo contenido de Se causan una baja actividad sanguínea de GSH-Px (Ceballos y Wittwer 1996). Así, la suplementación bajo diferentes formas de presentación de Se han inducido un incremento en la actividad de GSH-Px, ya que la actividad de la enzima está relacionada con el aporte del mineral (Wichtel 1998, Ceballos y col $\left.1999^{c}\right)$. En este estudio la suplementación con selenato de bario produjo una mayor actividad de GSH-Px, la que en el grupo Se-S fue mayor a partir del día 45 del estudio

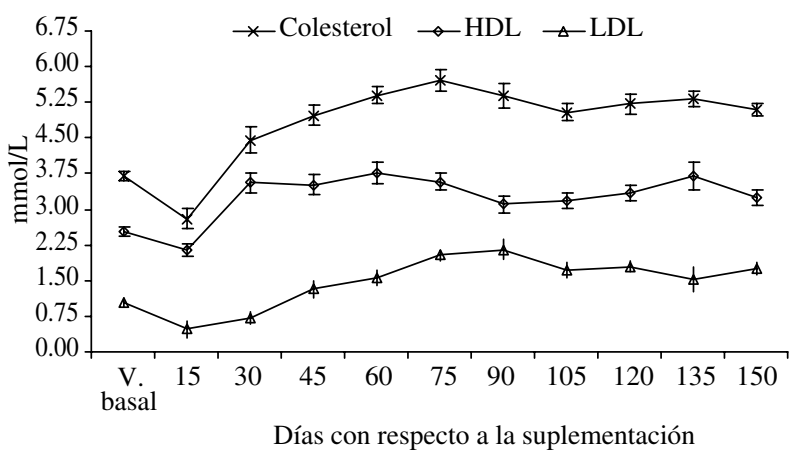

Figura 3. Concentración sérica $(\overline{\mathrm{X}} \pm \mathrm{EE})$ de colesterol, HDL y LDL de vacas lecheras alimentadas con una ración selenio deficiente o suplementadas con selenato de bario (se grafican los valores consolidados al no presentar diferencias estadísticamente significativas entre grupos).

Blood serum concentration $(\overline{\mathrm{X}} \pm \mathrm{SE})$ of cholesterol, HDL, and LDL of cows fed with a Se-deficient diet or supplemented with barium selenate (pooled values are shown since there was no significant difference between groups).

(figura 1). La actividad de la enzima aumenta después de la incorporación del mineral en los eritrocitos, proceso lento que conlleva un período de espera para evaluar el efecto de la suplementación por medio de la actividad de GSH-Px en sangre (Grace y col 2001).

Los resultados obtenidos en el presente trabajo concuerdan con otros estudios donde se han obtenido diferencias al suplementar con Se bajo la forma de selenato de bario, observándose que la actividad de la enzima se eleva a partir del día 46 después del tratamiento (Grace y col 2001). Asimismo, diferentes formas químicas de suplementación con Se han demostrado ser efectivas para inducir un aumento en la actividad de GSH-Px, lo que indica que estas formas químicas usadas para la suplementación se metabolizan en forma similar (Ortman y Pehrson 1999, Gunter y col 2003, Rowntree y col 2004).

Con respecto a la concentración de hormonas tiroideas, el bajo consumo de Se en la dieta puede inducir cambios en sus valores séricos en caprinos, bovinos y terneros (Wichtel y col 1996ab, Awadeh y col 1998, Rowntree y col 2004). Pese a lo anterior, no se ha demostrado que haya una interacción significativa entre el Se y el yodo, aunque la deficiencia del primero podría exacerbar el efecto de un bajo consumo de yodo (Wichtel y col 1996 ${ }^{\mathrm{ab}}$, Arthur y col 1999).

En este estudio las concentraciones de $\mathrm{T}_{3}$ fluctuaron dentro del rango de referencia $(0,8-2,0 \mathrm{nmol} / \mathrm{L})$ descrito por Burton (1992) e inferiores al señalado por Tiirats (1997). Los valores obtenidos para $T_{4}$ fueron inferiores al límite inferior referencial $(57-129 \mathrm{nmol} / \mathrm{L})$ señalado por Burton (1992), observándose algún grado de recuperación 
desde el día 90 del estudio en adelante sin lograr valores similares a los observados previamente al parto (figura 2). Sin embargo, la concentración de ambas hormonas fluctuó dentro del rango observado para vacas productoras de leche en el sur de Chile (Contreras y col 1999, Matamoros y col 2003, Contreras y col 2005).

La concentración de $\mathrm{T}_{3}$ no presentó diferencias entre los grupos a lo largo del estudio, mientras que los valores promedio de $\mathrm{T}_{4}$ fueron diferentes sólo para el valor basal (figura 2). En otros estudios se han descrito resultados diferentes, donde un bajo consumo de Se en la dieta para terneros condujo a una disminución en la concentración de $\mathrm{T}_{3}$ (Wichtel y col $1996^{\mathrm{b}}$ ) o un consumo adecuado en la ración para vacas (Awadeh y col 1998) causó incrementos en la concentración de $\mathrm{T}_{3} \sin$ afectar la de $\mathrm{T}_{4}$.

Las diferentes DI contienen residuos de selenocisteína en posiciones correspondientes a los codones UGA (TGA) en los respectivos ARN mensajeros (Allan y col 1999, Arthur y col 1999), lo que conlleva a un aumento de la actividad de las diferentes DI al suplementar con Se (Holben y Smith 1999, Bates y col 2000). La DI tipo I es responsable de la producción de dos terceras partes como mínimo de la $\mathrm{T}_{3}$ circulante derivada de la $\mathrm{T}_{4}$ (Brent 1994, Awadeh y col 1998, Arthur y col 1999), en un mecanismo donde el residuo de selenocisteína acepta la molécula de yodo en el centro activo de la enzima (Berry y Larsen 1992), originando un aumento de la concentración de $T_{3}$. Contrario a lo observado en este estudio, en otros reportes se ha observado una disminución significativa de la $\mathrm{T}_{4}$, efecto atribuido a una mayor deiodinación de la hormona; pero, no se encontraron cambios significativos en la concentración de $T_{3}$ (Wichtel y col 1996a).

Las concentraciones de hormonas tiroideas también se pueden ver afectadas por factores diferentes al contenido de Se en la dieta, como son el contenido de yodo en la ración, el consumo de grasas y el estado fisiológico de la vaca, entre otros (Grum y col 1996, Tiirats 1997, Arthur y col 1999, Contreras y col 1999, Matamoros y col 2003). Por lo tanto, su concentración obedece al resultado de múltiples factores y no se debería sólo al consumo de selenio.

Con respecto a los resultados del perfil lipídico se pudo establecer que no hubo diferencias significativas en las concentraciones de colesterol, lipoproteínas LDL y VLDL y TAG entre ambos grupos de vacas. En otros estudios se ha descrito el hallazgo de cambios en el metabolismo lipídico asociados con la concentración sérica de Se, observándose una elevación del colesterol y LDL en individuos con concentraciones bajas del mineral $(\mathrm{Qu}$ y col 2000, Huang y col 2002).

El grupo de vacas Se-D presentó una mayor concentración de HDL, encontrándose apenas una leve evidencia de significancia en los resultados $(\mathrm{P}=0,05)$. No obstante, la prueba estadística usada tuvo el suficiente poder (1-ß) para evitar errores en la prueba de significancia. Contrario a estos resultados, en roedores y mujeres se ha observado una concentración de HDL significativamente más baja en individuos con valores séricos de Se disminuidos $(\mathrm{Qu}$ y col 2000, Lee y col 2003). Lo anterior sería sugerente de la participación del Se en el metabolismo lipídico bien sea a través de un mecanismo directo o indirecto, pero esto requiere la realización de estudios posteriores; adicionalmente, no se encontraron referencias en la literatura internacional donde se haya evaluado el efecto de la ración con un bajo contenido de Se sobre la concentración de lipoproteínas en el bovino, lo que dificulta la comparación de los resultados con estudios similares.

El metabolismo de las lipoproteínas es un proceso complejo, donde unas se derivan de precursores aportados por otras lipoproteínas (Murray y col 2000). En el bovino el proceso de formación de las lipoproteínas es similar al descrito para los humanos con algunas variantes, especialmente en el fraccionamiento de estos compuestos (Bauchart 1993). La tendencia observada en este estudio podría estar asociada con una disminución en la síntesis de HDL, puesto que la actividad tiroidea regula la actividad de algunas de las enzimas que intervienen en el transporte de las lipoproteínas. Ha sido descrito (Duntas 2002) que la proteína que transfiere ésteres de colesterol y la lipasa hepática dependen del estado funcional de la tiroides, incrementando su actividad en los casos de elevaciones subclínicas de la concentración hormonal tiroidea, lo que conlleva a una reducción en la concentración de HDL.

Variaciones en el metabolismo de las lipoproteínas también están relacionadas con factores diferentes a la función tiroidea; así, se han descrito cambios asociados con el estado nutricional y productivo del rumiante (Bauchart 1993), balance energético (Grummer 1993, Drackley 1999), consumo de grasas en la dieta y los cambios asociados en la respuesta a estímulos hormonales mientras dure el uso de la grasa (Grum y col 1996, Drackley 1999), y con alteraciones circadianas en la síntesis o utilización lipídica independientes de cambios circadianos en los lípidos séricos (Bitman y col 1990).

Finalmente, la producción de leche está determinada por varios factores, entre otros se cuentan el estado sanitario general, la sanidad mamaria y la nutrición; pese a lo anterior, la suplementación con nutrientes específicos no siempre produce un incremento en la producción. Los resultados de este estudio concuerdan con lo anterior al no encontrarse diferencias para la producción de leche entre ambos grupos. En otros estudios se han obtenido resultados similares donde no se obtuvieron diferencias en la producción de leche al suplementar con Se en forma de bolos (Wichtel y col 1994) o como selenato de bario (Grace y col 2001).

En consideración a los resultados obtenidos, es posible señalar que el bajo consumo de Se manejado con la suplementación con selenato de bario no indujo cambios en la concentración de $T_{3}$, así como tampoco en la concentración de colesterol, lipoproteínas o TAG, observándose sólo una tendencia a una mayor concentración de lipoproteínas HDL en las vacas que recibieron una dieta baja en Se, mediante 
un mecanismo que no está completamente dilucidado en el bovino y cuya comprobación requiere de la realización de estudios posteriores con un mayor número de animales. Por el momento, la implicancia que tiene esta observación sobre el metabolismo lipídico del bovino sugiere que la suplementación con Se en animales eutiroideos puede producir una disminución en la concentración de HDL, afectando el metabolismo en el inicio de la lactancia o induciendo cambios en las funciones donde se requiere esta lipoproteína.

\section{RESUMEN}

El objetivo de este estudio fue evaluar el efecto de un bajo consumo de Se y su suplementación sobre la concentración sérica de $\mathrm{T}_{3}$ y $\mathrm{T}_{4} \mathrm{y}$ sobre el perfil lipídico en vacas lactantes bajo estrés oxidativo inducido. Fueron seleccionadas 12 vacas Frisón Negro gestantes, asignadas a uno de dos grupos homogéneos, control $(n=6, \mathrm{Se}-\mathrm{D})$ y tratado $(n=6, \mathrm{Se}-\mathrm{S})$ con selenato de bario $\left(\mathrm{BaSeO}_{4}\right)$, ambos recibieron una ración Se-deficiente (el Se en base seca fue 0,03 ppm para la dieta preparto y 0,05 ppm para la dieta posparto) y sebo oxidado $(0,5 \mathrm{~kg} / \mathrm{vaca} / \mathrm{día})$. El grupo $\mathrm{Se}-\mathrm{S}$ se suplementó con (1 mg Se/kg) bajo la forma de $\mathrm{BaSeO}_{4}(1 \mathrm{~mL} / 50 \mathrm{~kg}$, s.c.). Previo a la suplementación y cada 15 días, se tomaron muestras de sangre heparinizada y sin anticoagulante mediante venopunción coccígea. Se evaluó la actividad de glutatión peroxidasa (GSH-Px, EC 1.15.1.9), además la concentración sérica de $\mathrm{T}_{3}$ y $\mathrm{T}_{4}$, colesterol total, lipoproteínas (HDL, LDL y VLDL) y triacilgliceroles (TAG). La significancia se estableció empleando un PROC MIXED de SAS mediante la opción REPEATED, considerándose significativo $\mathrm{P}<0,05$. La suplementación con $\mathrm{BaSeO}_{4}$ no produjo cambios en la producción de leche entre los grupos $(\mathrm{P}>0,05)$, y la actividad de GSH-Px aumentó significativamente en el grupo Se-S a partir de 45 días postsuplementación $(\mathrm{P}<0,05)$, la actividad en el grupo Se-D se mantuvo baja a lo largo del estudio $(\mathrm{P}<0,05)$. No hubo diferencias para $\mathrm{T}_{3}$ y $_{4}(\mathrm{P}>0,05)$. La concentración de colesterol, LDL, VLDL y TAG fue similar entre los grupos $(\mathrm{P}>0,05)$, siendo la concentración de HDL levemente mayor en el grupo Se-D $(\mathrm{P}=0,047)$. Los resultados permiten señalar que una dieta baja en Se no indujo cambios en la función tiroidea o en el metabolismo de las lipoproteínas, excepto una mayor concentración de HDL, lo que en el bovino requiere la realización de estudios posteriores.

\section{AGRADECIMIENTOS}

Al Dr. Henry Mesa por sus amables sugerencias para el mejoramiento en el análisis de los resultados.

\section{REFERENCIAS}

Allan CB, GM Lacourciere, TC Stadtman. 1999. Responsiveness of selenoproteins to dietary selenium. Annu Rev Nutr 19, 1-16.

Arthur JR, GJ Beckett, JH Mitchell. 1999. The interactions between selenium and iodine deficiencies in man and animals. Nutr Res Rev 12, 55-73.

Awadeh FT, RL Kincaid, KA Johnson. 1998. Effect of level and source of dietary selenium on concentrations of thyroid hormones and immunoglobulins in beef cows and calves. J Anim Sci 76, 1204-1215.

Bates JM, VL Spate, JS Morris, DL St Germain, VA Galton. 2000. Effects of selenium deficiency on tissue selenium content, deiodinase activity, and thyroid hormone economy in the rat during development. Endocrinology 141, 2490-2500.

Bauchart D. 1993. Lipid absorption and transport in ruminants. J Dairy Sci 76, 3864-3881.

Berry MJ, PR Larsen. 1992. The role of selenium in thyroid hormone action. Endocr Rev 13, 207-219.
Bitman J, DL Wood, AM Lefcoufil. 1990. Rhythms in cholesterol, cholesteryl esters, free fatty acids, and triglycerides in blood of lactating dairy cows. J Dairy Sci 73, 948-955.

Brent GA. 1994. The molecular basis of thyroid hormone action. $N$ Engl J Med 331, 847-853.

Burton S. 1992. Handbook of diagnostic endocrinology. Atlantic Veterinary College, University of Prince Edward Island, Charlottetown, Canada.

Ceballos A, F Wittwer. 1996. Metabolismo del selenio en rumiantes. Arch Med Vet 28, 5-18.

Ceballos A, F Wittwer, PA Contreras, H Böhmwald. 1998ª . Actividad sanguínea de glutatión peroxidasa en rebaños lecheros a pastoreo: Variación según edad y época del año. Arch Med Vet 30, 13-22.

Ceballos A, A Patitucci, O Romero, M Andaur, P Asenjo. 1998 ${ }^{\text {b }}$. Miopatía nutricional por deficiencia de selenio en caprinos de una explotación en la IX Región, Chile. XXIII Reunión Anual de la Sociedad Chilena de Producción Animal, Chillán, Chile.

Ceballos A, F Wittwer, O Romero, M Andaur. 1999ª . Actividad de glutatión peroxidasa y ganancia de peso en cabras Cashmere y mestizas suplementadas con selenio. XXIV Reunión Anual de la Sociedad Chilena de Producción Animal, Temuco, Chile.

Ceballos A, R Matamoros, F Wittwer, O Romero, M Andaur. 1999 Efecto de la suplementación con selenio sobre la concentración de tiroxina y la ganancia de peso en cabras Cashmere y Cashmere por criollo. XXIV Reunión Anual de la Sociedad Chilena de Producción Animal, Temuco, Chile.

Ceballos A, FG Wittwer, PA Contreras, E Quiroz, H Böhmwald. 1999 Actividad de glutatión peroxidasa en bovinos lecheros a pastoreo correlacionada con la concentración sanguínea y plasmática de selenio. Pesq agropec bras 34, 2331-2338.

Contreras PA, F Wittwer, V Ruiz, A Robles, H Böhmwald. 1999. Valores sanguíneos de triyodotironina y tiroxina en vacas frisón negro a pastoreo. Arch Med Vet 31, 205-210.

Contreras PA, R Matamoros, R Monroy, J Kruze, V Leyán, M Andaur, H Böhmwald, F Wittwer. 2002. Effect of a selenium-deficient diet on blood values of T3 and T4 in cows. Comp Clin Path 11, 65-70.

Contreras PA, F Wittwer, R Matamoros, IM Mayorga, G Van Schaik. 2005. Effect of grazing pasture with a low selenium content on the concentrations of tri-iodothyronine and thyroxine in serum, and GSH-Px activity in erythrocytes in cows in Chile. New Zeal Vet J 53, 77-80.

Drackley JK. 1999. Biology of dairy cows during the transition period: the final frontier? J Dairy Sci 82, 2259-2273.

Duntas LH. 2002. Thyroid disease and lipids. Thyroid 12, 287-293.

Grace ND, K Ankebauer-Perkins, AM Alexander, RM Marchant. 2001. Relationship between blood selenium concentration or glutathione peroxidase activity, and milk selenium concentrations in New Zealand dairy cows. New Zeal Vet J 49, 25-29.

Grum DE, JK Drackley, RS Younker, DW Lacount, JJ Veenhuizen. 1996. Nutrition during the dry period and hepatic lipid metabolism of periparturient dairy cows. J Dairy Sci 79, 1850-1864.

Grummer RR. 1993. Etiology of lipid-related metabolic disorders in periparturient dairy cows. J Dairy Sci 76, 3882-3896.

Gunter SA, PA Beck, JM Phillips. 2003. Effects of supplementary selenium source on the performance and blood measurements in beef cows and their calves. J Anim Sci 81, 856-864.

Holben DH, AM Smith. 1999. The diverse role of selenium within selenoproteins: A review. J Amer Diet Assoc 99, 836-843.

Howie AF, SW Walker, B Åkesson, JR Arthur, GJ Beckett. 1995. Thryroidal extracellular glutathione peroxidase: A potential regulator of thyroid-hormone synthesis. Biochem J 308, 713-717.

Huang K, H Liu, Z Chen, H Xu. 2002. Role of selenium in cytoprotection against cholesterol oxide-induced vascular damage in rats. Atherosclerosis 162, 137-144.

Lee O, J Moon, Y Chung. 2003. The relationship between serum selenium levels and lipid profiles in adult women. J Nutr Sci Vitaminol (Tokyo) 49, 397-404. 
Matamoros R, PA Contreras, F Wittwer, MI Mayorga. 2003. Hipotiroidismo en rumiantes. Arch Med Vet 35, 1-11.

Miller JK, E Brzezinska-Slebodzinska, FC Madsen. 1993. Oxidative stress, antioxidants, and animal function. J Dairy Sci 76, 2812-2823.

Murray RK, DK Granner, PA Mayes, VW Rodwell. 2000. Harper's biochemistry. $25^{\text {th }}$ ed. Appleton \& Lange, Stamford, USA.

Ortman K, B Pehrson. 1999. Effect of selenate as a feed supplement to dairy cows in comparison to selenite and selenium yeast. J Anim Sci 77, 3365-3370.

Qu X, K Huang, L Deng, H Xu. 2000. Selenium deficiency-induced alterations in the vascular system of the rat. Biol Trace Elem Res 75, 119-128.

Rowntree JE, GM Hill, DR Hawkins, JE Link, MJ Rincker, GW Bednar, RA Kreft Jr. 2004. Effect of Se on selenoprotein activity and thyroid hormone metabolism in beef and dairy cows and calves. J Anim Sci 82, 2995-3005.

Ruz M, J Codoceo, J Galgani, L Muñoz, N Gras, S Muzzo, L Leiva, C Bosco. 1999. Single and multiple selenium-zinc-iodine deficiencies affect rat thyroid metabolism and ultrastructure. J Nutr 129, 174-180.

Tiirats T. 1997. Thyroxine, triiodothyronine and reverse-triiodothyronine concentrations in blood plasma in relation to lactational stage, milk yield, energy and dietary protein intake in Estonian dairy cows. Acta Vet Scand 38, 339-348.

Wichtel JJ, AL Craigie, H Varela-Álvarez, NB Williamson. 1994. The effect of intra-ruminal selenium pellets on growth rate, lactation and reproductive efficiency in dairy cattle. New Zeal Vet J 42, 205-210.

Wichtel JJ, KG Thompson, AL Craigie, NB Williamson. 1996 ${ }^{\mathrm{a}}$. Effects of selenium and iodine supplementation on the growth rate, mohair production, and thyroid status of Angora goat kids. New Zeal J Agr Res 39, 111-115.

Wichtel JJ, AL Craigie, DA Freeman, H Varela-Álvarez, NB Williamson. $1996^{\text {b }}$. Effects of selenium and iodine supplementation on growth rate and on thyroid and somatotropic function in dairy calves at pasture. J Dairy Sci 79, 1865-1872.

Wichtel JJ. 1998. A review of selenium deficiency in grazing ruminants. Part 1: New roles for selenium in ruminant metabolism. New Zeal Vet $J$ 46, 47-52.

Wittwer F, P Araneda, A Ceballos, PA Contreras, MAndaur, H Böhmwald. 2002. Actividad de glutatión peroxidasa (GSH-Px) en sangre de bovinos a pastoreo de la IX Región, Chile y su relación con la concentración de selenio en el forraje. Arch Med Vet 34, 49-57. 